\title{
Characterization of Non-endcapped Polymeric ODS Column for the Separation of Triacylglycerol Positional Isomers
}

\author{
Naohiro Gotoh${ }^{1}$, Yumiko Matsumoto', Hiromi Yuji ${ }^{1}$, Toshiharu Nagai², Hoyo Mizobe ${ }^{2}$, \\ Kenji Ichioka ${ }^{2}$, Ikuma Kuroda ${ }^{3}$, Noriko Noguchi ${ }^{4}$, and Shun Wada ${ }^{1 *}$ \\ ${ }_{1}^{1}$ Department of Food Science and Technology, Tokyo University of Marine Science and Technology (4-5-7 Konan, Minato-ku, Tokyo 108-8477, JAPAN) \\ ${ }^{2}$ Tsukishima Foods Industry Co. Ltd. (3-17-9, Higashi Kasai,Edogawa-ku,Tokyo 134-8520, JAPAN) \\ ${ }^{3}$ GL Sciences Inc. (237-2 Sayamagahara, Iruma, Saitama 358-0032, JAPAN) \\ ${ }^{4}$ Department of Medical Life Systems, Doshisha University (1-3 Tatara Miyakodani, Kyotanabe, Kyoto 610-0394, JAPAN)
}

\begin{abstract}
The characteristics of a non-endcapped polymeric ODS column for the resolution of triacylglycerol positional isomers (TAG-PI) were examined using a recycle HPLC-atmospheric pressure chemical ionization/mass spectrometry system. A pair of TAG-PI containing saturated fatty acids at least 12 carbons was separated. Except for TAG-PI containing elaidic acid, pairs of TAG-PI containing three unsaturated fatty acids were not separated, even by recycle runs. These results indicate that the resolution of TAG-PI on a non-endcapped polymeric ODS stationary phase is realized by the recognition of the linear structure of the fatty acid and the binding position of the saturated fatty acid in TAG-PI. Chain length was also an important factor for resolution. This method may be a useful and simple for measuring the abundance ratio of TAG-PI containing saturated fatty acids in natural oils.
\end{abstract}

Key word: polymeric ODS column, positional isomer, recycle HPLC system, triacylglycerol

\section{INTRODUCTION}

Triacylglycerol (TAG) is the most abundant class of lipids in nature. TAG comprises one glycerol and three fatty acids and all the fatty acids are esterified with alcohol groups in the glycerol ${ }^{11}$. Since many kinds of fatty acids exist in nature, the three fatty acids in TAG have an almost infinite number of combinations. The TAGs consisted of different kinds of fatty acids are recognized as different kind of TAG molecular species. TAGs are further distinguished by the binding position, either alpha $(\alpha)$ or beta $(\beta$, of the fatty acids on the glycerol backbone ${ }^{2}$. Two kinds of alcohol groups, primary and secondary, are contained in glycerol. The primary and secondary alcohol groups are also discriminated by their position, $\alpha$ and $\beta$, respectively. Pancreatic lipase is an enzyme that hydrolyzes fatty acids located at the $\alpha$ position of TAG in small intestine to form two fatty acids and 2-monoacylglycerol ${ }^{3}$. TAG comprising two kinds of fatty acids, "A" and "B", with two A and one B on the glycerol backbone is possible to make two kinds of TAG molecular species, i.e., TAG with A locating at the $\beta$ position ( $\beta$-AAB) and TAG with B locating at the $\beta$ position $(\beta-\mathrm{ABA})^{2)}$. Though these TAGs consist of same kinds and numbers of fatty acids, they are considered as TAG positional isomers (TAG-PI). The characteristics of TAG regarding digestion, absorption, and physiology are deeply related to its structure, namely the TAG molecular species and TAG-PI abundance ratio ${ }^{4-6}$.

Various methods to separate and quantify TAG-PI pairs have been developed. Silver ion chromatography is the most well-known and popular method of separating TAGPI pairs ${ }^{7-9)}$. In general, the separation of TAG-PI pairs by silver ion chromatography is not easy because of their low reproducibility. Furthermore, the lifetime of the silver ion

Abbreviations: APCI, atmospheric pressure chemical ionization; $\mathrm{C}_{8}$, caprylic acid; $\mathrm{C}_{10}$, capric acid; $\mathrm{C}_{12}$, lauric acid; $\mathrm{C}_{14}$, myristic acid; $\mathrm{C}_{16}$, palmitic acid; $\mathrm{C}_{18}$, stearic acid; $\mathrm{D}, \mathrm{DHA}$; $\mathrm{DAG}^{+}$, positive diacylglycerol peak; El, elaidic acid; MS, mass spectrometry; O, oleic acid; Rs, resolution factor; TAG, triacylglycerol; TAG-PI, triacylglycerol positional isomer; $\mathrm{TF}$, tailing factor

\footnotetext{
*Correspondence to: Shun Wada, Department of Food Science and Technology, Tokyo University of Marine Science and Technology, 4-57 Konan, Minato-ku, Tokyo 108-8477, JAPAN

E-mail: wada@kaiyodai.ac.jp

Accepted October 15, 2009 (received for review August 3, 2009)
}

Journal of Oleo Science ISSN 1345-8957 print / ISSN 1347-3352 online

http://www.jstage.jst.go.jp/browse/jos/ 
column is short due to the fact that TAG-PI is eluted by $n$ hexane mixed with a small amount of acetonitrile, which is a polar organic compound and the silver is therefore gradually eliminated from the matrix of the column. The elimination of the silver also impairs detection of the sample because the silver ion eluted from the column appears as noises on the chromatogram. HPLC/atmospheric pressure chemical ionization (APCI)-mass spectrometry (MS) is also used to quantify the TAG-PI abundance ratio ${ }^{10-14)}$. Mottram et al. developed an HPLC/APCI-MS method to easily distinguish the binding position, $\alpha$ or $\beta$, of the fatty acids in TAG molecular species ${ }^{12}$. In this method, each TAG-PI is not separated on the chromatogram. The fatty acid locating at the $\alpha$ position is relatively easy to cleave under APCI compared to that at the $\beta$ position. Therefore, the peak area of the positive diacylglycerol peak $\left(\mathrm{DAG}^{+}\right)$formed by cleaving off fatty acid moiety locating at the $\beta$ position is smaller than that formed by fatty acid located at the $\alpha$ position. A calibration curve, made by plotting the abundance ratio of TAG-PI on the $\mathrm{x}$-axis and the relative peak area of the two kinds of $\mathrm{DAG}^{+}$formed under the APCI conditions on the y-axis, can be used to quantify the TAG-PI abundance ratio. Although this method has been adapted to the analysis of TAG-PI in plant oils and animal fats ${ }^{13-15}$, it cannot be used directly to quantify the TAG-PI abundance ratio and a calibration curve must be prepared for the individual TAG-PI pair before beginning the chromatography because the slope of the calibration curve differs among the TAG-PI pairs. Thus, a simple, rapid, and with high reproducible TAG-PI analytic method is desired.

Recently, Momchilova et al. reported the separation of TAG-PI pairs using a non-endcapped ODS column ${ }^{16,17}$. They reported good resolution for six different TAG-PI pairs comprising two palmitic acids and one unsaturated fatty acid. Kuroda et al. also demonstrated that a non-endcapped polymeric ODS column can separate TAG-PI pairs consisting of two palmitic acids and one oleic acid or one palmitic acid and two oleic acids ${ }^{18}$. These methods are very simple and reproducible and may be useful for the separation of TAG-PI. All of these previous studies with ODS columns used TAG-PI pairs containing one or two palmitic acids to confirm the separations. Therefore, characterization of a non-endcapped polymeric ODS column for the separation of TAG-PI pairs is important to evaluate the usefulness and effectiveness of this method. In the present study, we examined the resolution of several kinds of TAGPI pairs using a recycle HPLC/APCI-MS system equipped with a non-endcapped polymeric ODS column.

\section{EXPERIMENTAL}

\subsection{Chemicals and materials}

The TAG-PIs shown in Table 1 were obtained from
Tsukishima Food Industry Co., Ltd (Tokyo, Japan). All other reagents were purchased from Wako Pure Chemical Industries, Ltd. (Osaka, Japan).

\subsection{Analytical conditions}

The same amount of TAG-PI pairs was dissolved in ethanol and adjusted to approximately $20 \mathrm{ppm}$. The samples were subjected to a recycle HPLC-APCI/MS system consisting of a pump (PU-611C, GL Sciences Inc., Tokyo, Japan), a UV-Vis detector (UV-702, GL Sciences Inc.), a column oven (MO-706, GL Sciences Inc.), a recycle valve (HPV-Rc, GL Sciences Inc.), an injection valve equipped with a 50- $\mu \mathrm{L}$ sample loop (Injector: Model 7725, Rheodyne LLC, Rohnert Park, CA), an APCI-MS (Waters Alliance ZMD LC/MS System, Waters Corporation, Milford, MA), and tandem jointed non-endcapped polymeric ODS columns (Inertsil ODS-P; $\alpha \mathrm{TBN} / \mathrm{BaP}=0.7,29 \%$ carbon content, 250 x 4.6 mm, i.d. $5 \mu \mathrm{m}$; GL Sciences Inc.). A mixture of acetonitrile and 2-propanol $(6: 4, \mathrm{v} / \mathrm{v})$ was used as the mobile phase for the separation of TAG-PI. The flow rate was $0.8 \mathrm{~mL} / \mathrm{min}$ and the injection volume was $20 \mu \mathrm{L}$. The column temperatures were $30^{\circ} \mathrm{C}$ for TAG-PI containing stearic acid $\left(\mathrm{C}_{18}\right), 22^{\circ} \mathrm{C}$ for TAG-PI containing palmitic acid $\left(\mathrm{C}_{16}\right)$, and $18^{\circ} \mathrm{C}$ for the other kinds of TAG-PIs. The recycle system was used only when the TAG-PI pair was not separated. Data acquisition, processing, and instrument control of the APCI-MS were performed using MassLynx software (Waters Corporation). The APCI conditions were corona voltage $4.0 \mathrm{kV}$, positive-mode cone voltage 40 $\mathrm{V}$, negative-mode cone voltage $-40 \mathrm{~V}$, heater temperature $400^{\circ} \mathrm{C}$, and nitrogen gas flow $500 \mathrm{~L} / \mathrm{h}$. The analyses were performed in positive ion mode. Spectra were obtained over the range of $m / z$ 500-700 and $m / z$ 800-1050 for TAGPI containing two caprylic acids $\left(\mathrm{C}_{8}\right)$ and two DHAs (D), respectively, with a scan time $0.4 \mathrm{~s}$. TAG-PIs were monitored at $m / z \mathrm{M}+18$, namely by the formation of ammonium adduct ion $\left(\mathrm{NH}_{4}{ }^{+}\right)$, because the intensity of ammonium adduct ion was stronger than the proton adduct ion in our analytical condition. This might be due to the existence of acetonitrile in the mobile phase; however, the details are not clear. The detection of TAG-PIs containing DHA was also carried out using the UV-Vis detector at $210 \mathrm{~nm}$. In this experiment, APCI/MS was used for the detection of TAG-PIs because TAG-PIs comprising three saturated fatty acids do not have characteristic UV absorbance like DHA, and for this reason it is not possible to monitor and confirm the separation of TAG-PI pairs consisting of three saturated fatty acids using a recycle system with a UV-Vis detector.

\subsection{Evaluation of the resolution and tailing of the peaks}

Resolution factor (Rs) was calculated from the following equation ${ }^{19,20)}$. 
Table 1 Structures of TAG-PI Used in This Study.

\begin{tabular}{|c|c|c|c|}
\hline$\beta-\mathrm{C}_{8} \mathrm{C}_{8} \mathrm{C}_{10}$ & $B-C_{8} C_{10} C_{8}$ & $ß-\mathrm{DDC}_{10}$ & $\beta-\mathrm{DC}_{10} \mathrm{D}$ \\
\hline$\beta-\mathrm{C}_{8} \mathrm{C}_{8} \mathrm{C}_{12}$ & $\beta-C_{8} C_{12} C_{8}$ & $\beta-\mathrm{DDC}_{12}$ & $\beta-\mathrm{DC}_{12} \mathrm{D}$ \\
\hline$\beta-\mathrm{C}_{8} \mathrm{C}_{8} \mathrm{C}_{14}$ & $\beta-\mathrm{C}_{8} \mathrm{C}_{14} \mathrm{C}_{8}$ & $\beta-\mathrm{DDC}_{14}$ & $\beta-\mathrm{DC}_{14} \mathrm{D}$ \\
\hline$\beta-\mathrm{C}_{8} \mathrm{C}_{8} \mathrm{C}_{16}$ & $\beta-C_{8} C_{16} C_{8}$ & $\beta-\mathrm{DDC}_{16}$ & $\beta-\mathrm{DC}_{16} \mathrm{D}$ \\
\hline$B-\mathrm{C}_{8} \mathrm{C}_{8} \mathrm{C}_{18}$ & $\beta-\mathrm{C}_{8} \mathrm{C}_{18} \mathrm{C}_{8}$ & $\beta-\mathrm{DDC}_{18}$ & $\beta-\mathrm{DC}_{18} \mathrm{D}$ \\
\hline \multirow[t]{2}{*}{$B-\mathrm{C}_{8} \mathrm{C}_{8} \mathrm{D}$} & $\beta-\mathrm{C}_{8} \mathrm{DC}_{8}$ & ß-DDO & $\beta-\mathrm{DOD}$ \\
\hline & & $\beta$-DDE1 & $\beta-D E 1 D$ \\
\hline
\end{tabular}

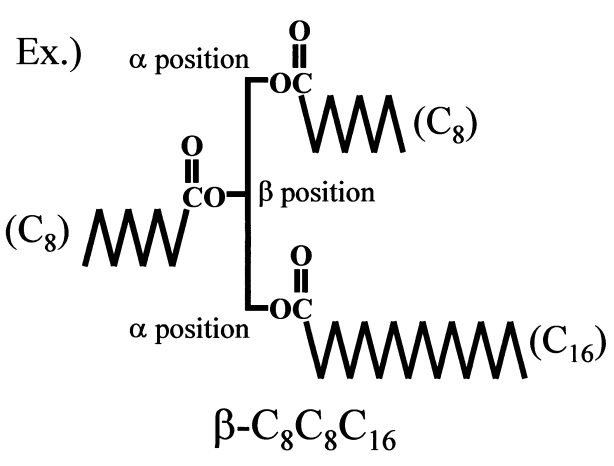

This TAG has a fixed $C_{8}$ at the $\beta$ position and other fatty acids are located at the $\alpha$ position. In this case, the TAG is expressed as $\beta-C_{8} C_{8} C_{16}$.

\section{$M^{\mathrm{CoOH}}$}

Caprylic acid $\left(\mathrm{C}_{8}\right)$

$\mathrm{MWW}^{\text {соон }}$

Palmitic acid $\left(\mathrm{C}_{16}\right)$

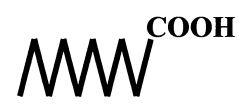

Capric acid $\left(\mathrm{C}_{10}\right)$

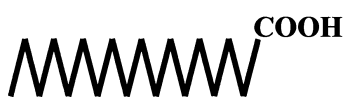

Stearic acid $\left(\mathrm{C}_{18}\right)$

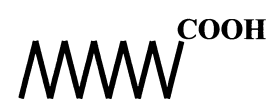

Lauric acid $\left(\mathrm{C}_{12}\right)$

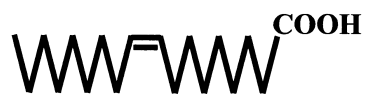

Oleic acid $\left(\right.$ cis- $\left.\mathrm{C}_{18: 1}: \mathrm{O}\right)$

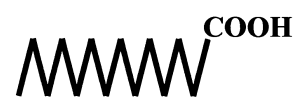

Myristic acid $\left(\mathrm{C}_{14}\right)$

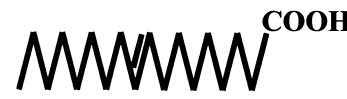

Elaidic acid (trans- $\left.\mathrm{C}_{18: 1}: \mathrm{El}\right)$

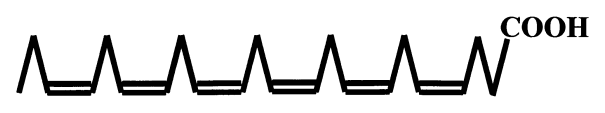

\section{Docosahexaenoic acid (DHA:D) \\ Notation of abbreviation in Table 1}

$$
\mathrm{Rs}_{\mathrm{S}}=2\left(\mathrm{t}_{\mathrm{R} 2}-\mathrm{t}_{\mathrm{R} 1}\right) /\left(\mathrm{W}_{1}+\mathrm{W}_{2}\right),
$$

where $t_{R 1}$ and $t_{R 2}$ are the retention times and $W_{1}$ and $W_{2}$ are the widths of the first- and second-eluted peaks, respectively. In general, separation is evaluated as follows; $98 \%$ resolution: $\mathrm{Rs}=1.00$, baseline resolution $(99.5 \%$ resolution): $\mathrm{Rs}_{\mathrm{s}}=1.25$, and complete resolution: $\mathrm{Rs}>1.50$.

Tailing factor $(\mathrm{TF})$ was calculated from the following equation ${ }^{20)}$.

$$
\mathrm{TF}=(\mathrm{A}+\mathrm{B}) / 2 \mathrm{~A},
$$

where $\mathrm{A}$ and $\mathrm{B}$ are the peak widths of the left and rightsides, respectively, at the $5 \%$ height of the peak when the peak was separated by a line segment passing through the top of the peak and perpendicular to the baseline. The peak was considered symmetrical when TF was 1.00.

Analyses of each TAG-PI pair were carried out in triplicate and then the mean Rs and TF were calculated.

\section{RESULTS}

Chromatograms showing the resolution of TAG-PIs containing two $\mathrm{C}_{8}$ are shown in Fig. 1. No resolution was observed for TAG-PI pairs containing capric acid $\left(\mathrm{C}_{10}\right)$ or D, even when using the recycle system. TAG-PI pairs comprising two $\mathrm{C}_{8}$ and one lauric acid $\left(\mathrm{C}_{12}\right)$ were separated by two recycle runs. Other kinds of TAG-PIs were separated by a non-endcapped polymeric ODS column without requiring a recycle run. Mean Rs and TF for TAG-PI pairs containing two $\mathrm{C}_{8} \mathrm{~S}$ are summarized in Table 2. The mean Rs was greater than 1.00 for all three separated TAG-PIs and the mean TF was less than 2.00. Similarly, chromatograms showing the resolution of TAG-PIs containing two Ds are also shown in Fig. 2 and the mean Rs and mean TF are summarized in Table 3. Although a TAG-PI consisting of two Ds and one $\mathrm{C}_{12}$ could be resolved in this case, the resolution was poor $\left(\mathrm{Rs}_{\mathrm{S}}=0.22\right)$. Other resolution characteristics of TAG-PI were similar between two kinds TAG-PI families. The mean TF values for TAG-PI comprising two Ds with one $\mathrm{C}_{16}$ or two Ds with one $\mathrm{C}_{18}$ were 2.00 or higher. The separation of TAG-PI comprising two Ds with one 


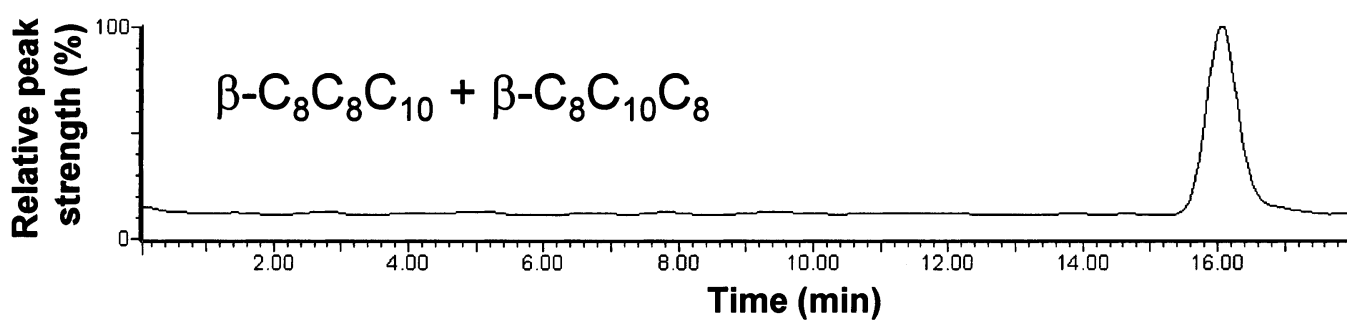

at $18^{\circ} \mathrm{C}$

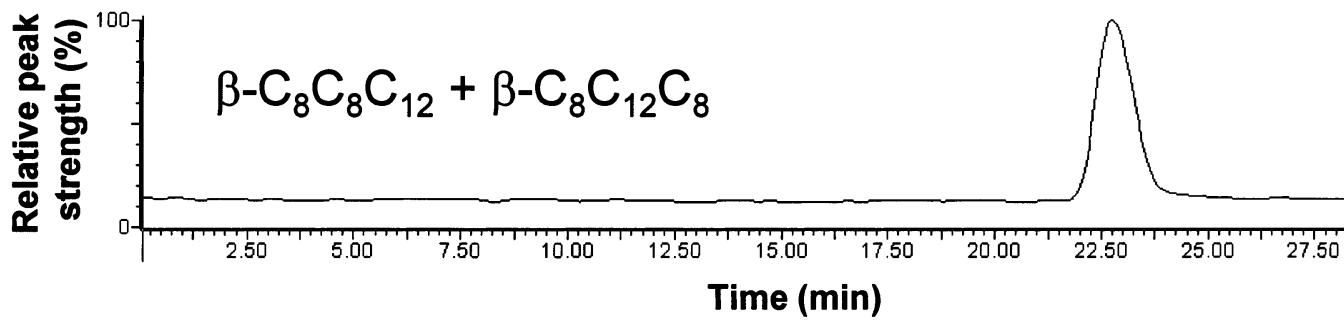

at $18^{\circ} \mathrm{C}$

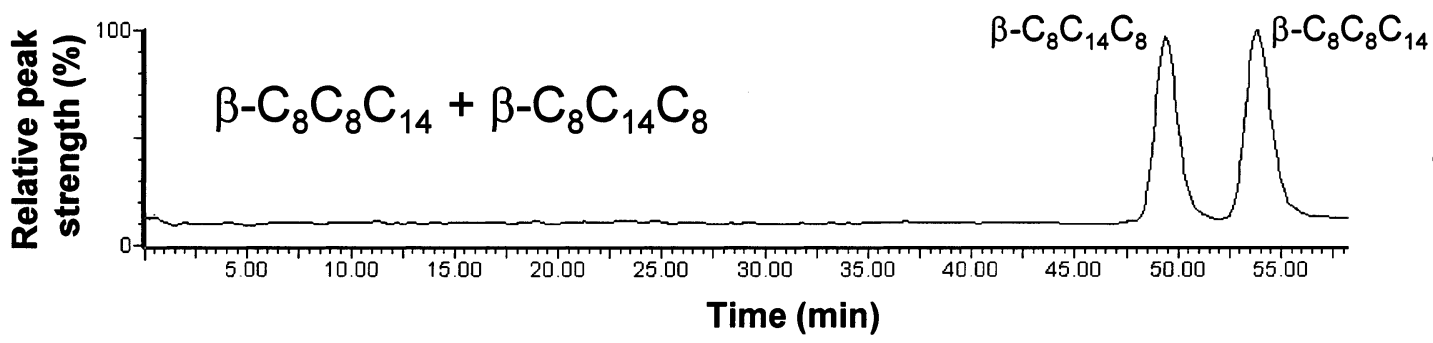

at $18^{\circ} \mathrm{C}$



at $22^{\circ} \mathrm{C}$

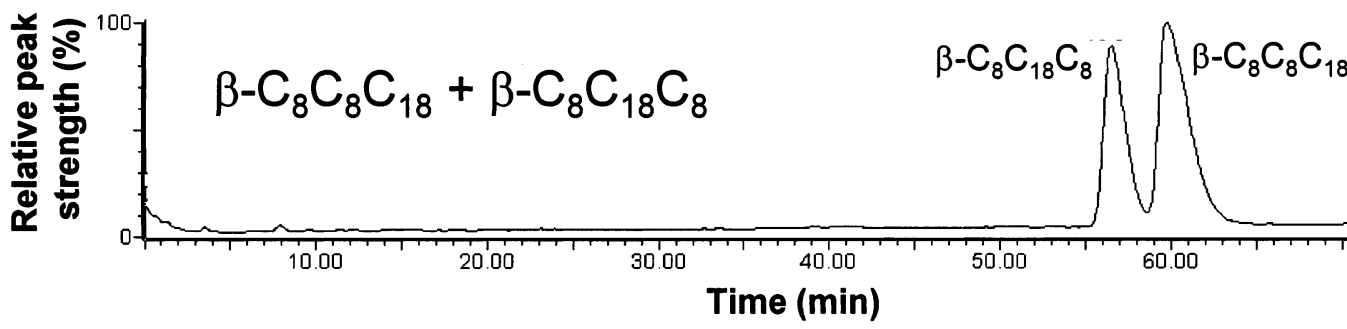

at $30^{\circ} \mathrm{C}$



at $18^{\circ} \mathrm{C}$

Fig. 1 Comparison of the Resolution of TAG-PI Pairs Containing Two $\mathrm{C}_{8} \mathrm{~s}$ on Polymeric ODS Column.

$\mathrm{D}$ designates docosahexaenoic acid moiety. 


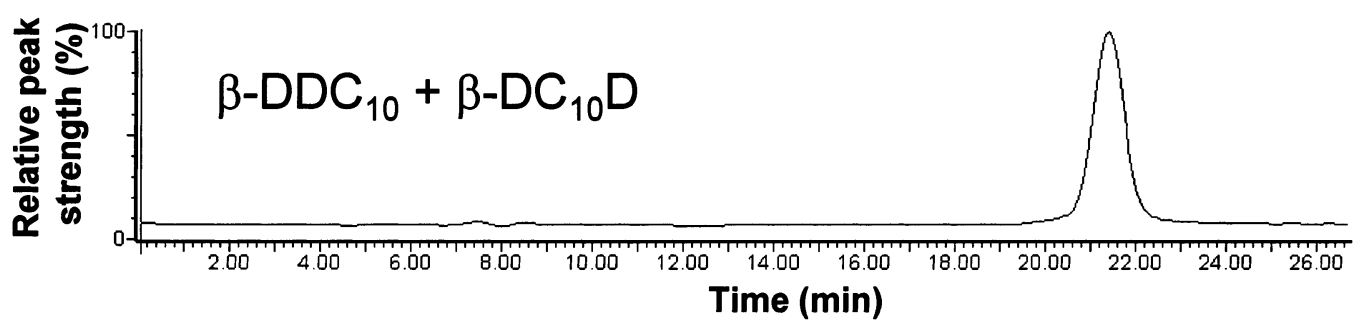

at $18^{\circ} \mathrm{C}$

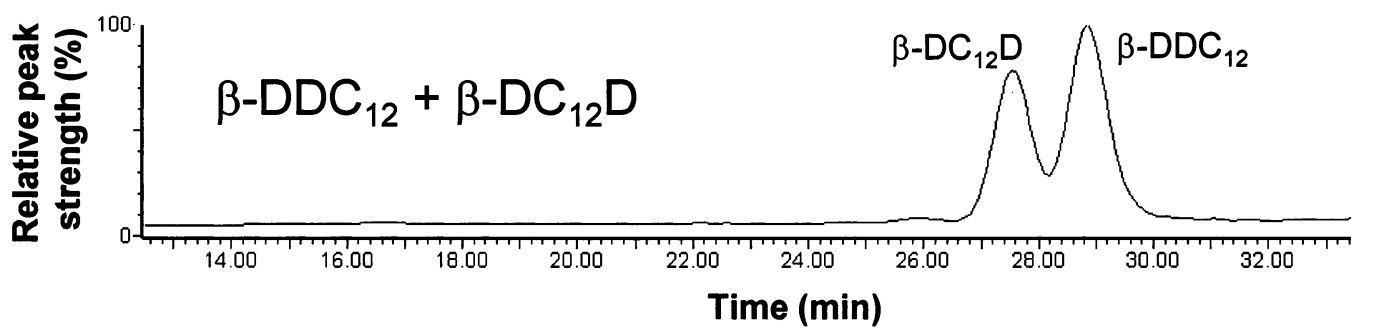

at $18^{\circ} \mathrm{C}$

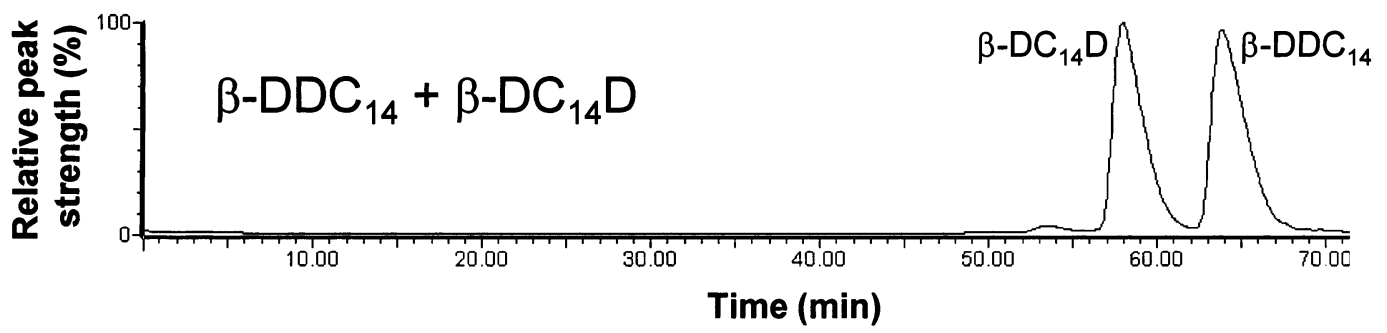

at $18^{\circ} \mathrm{C}$

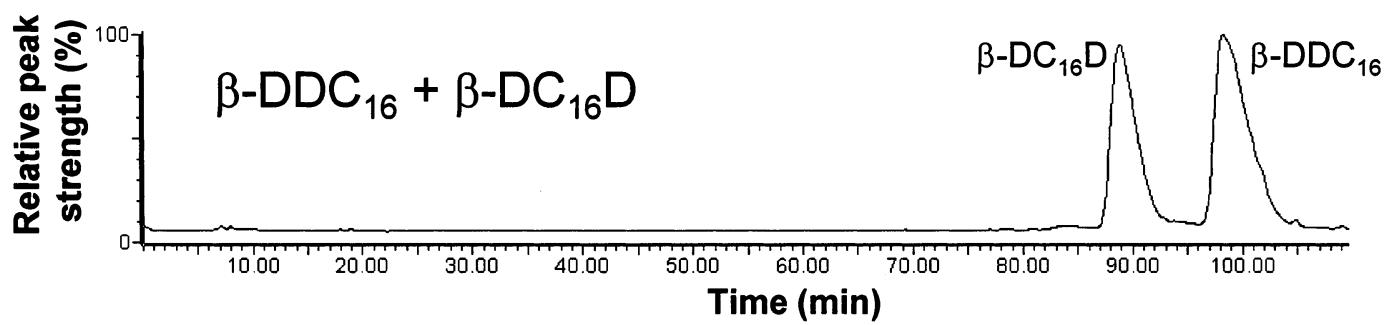

at $22^{\circ} \mathrm{C}$

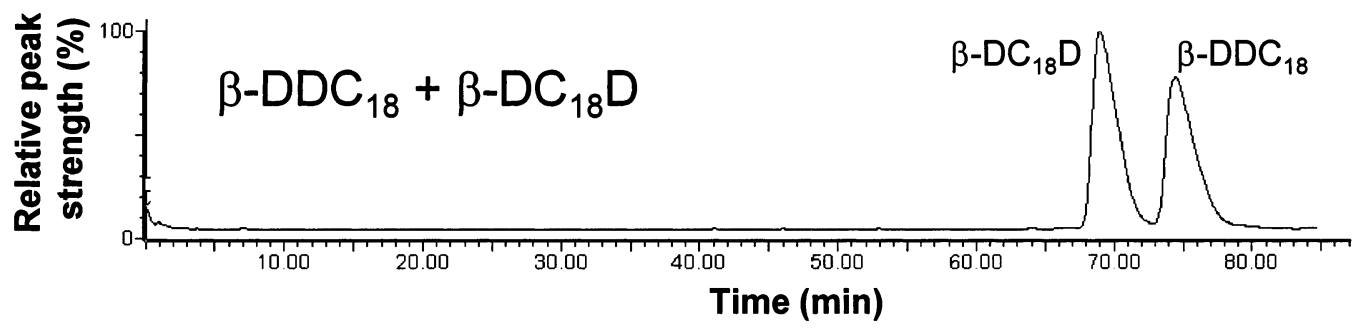

at $30^{\circ} \mathrm{C}$

Fig. 2 Comparison of the Resolution of TAG-PI Pairs Containing Two Ds on Polymeric ODS Column. D designates docosahexaenoic acid moiety.

monounsaturated fatty acid was also compared using the recycle system (Fig. 3). When TAG-PI containing oleic acid (O), a monounsaturated fatty acid with a cis-type double bond, was compared with TAG-PI containing elaidic acid (El), a monounsaturated fatty acid with a trans-type double bond, separation of the TAG-PI pair containing $\mathrm{O}$ was not achieved, even after more than 10 recycle runs, while sepa- ration of a TAG-PI pair containing El was possible after approximately 5 recycle runs (the separated peak is indicated by the arrow in Fig. 3). 

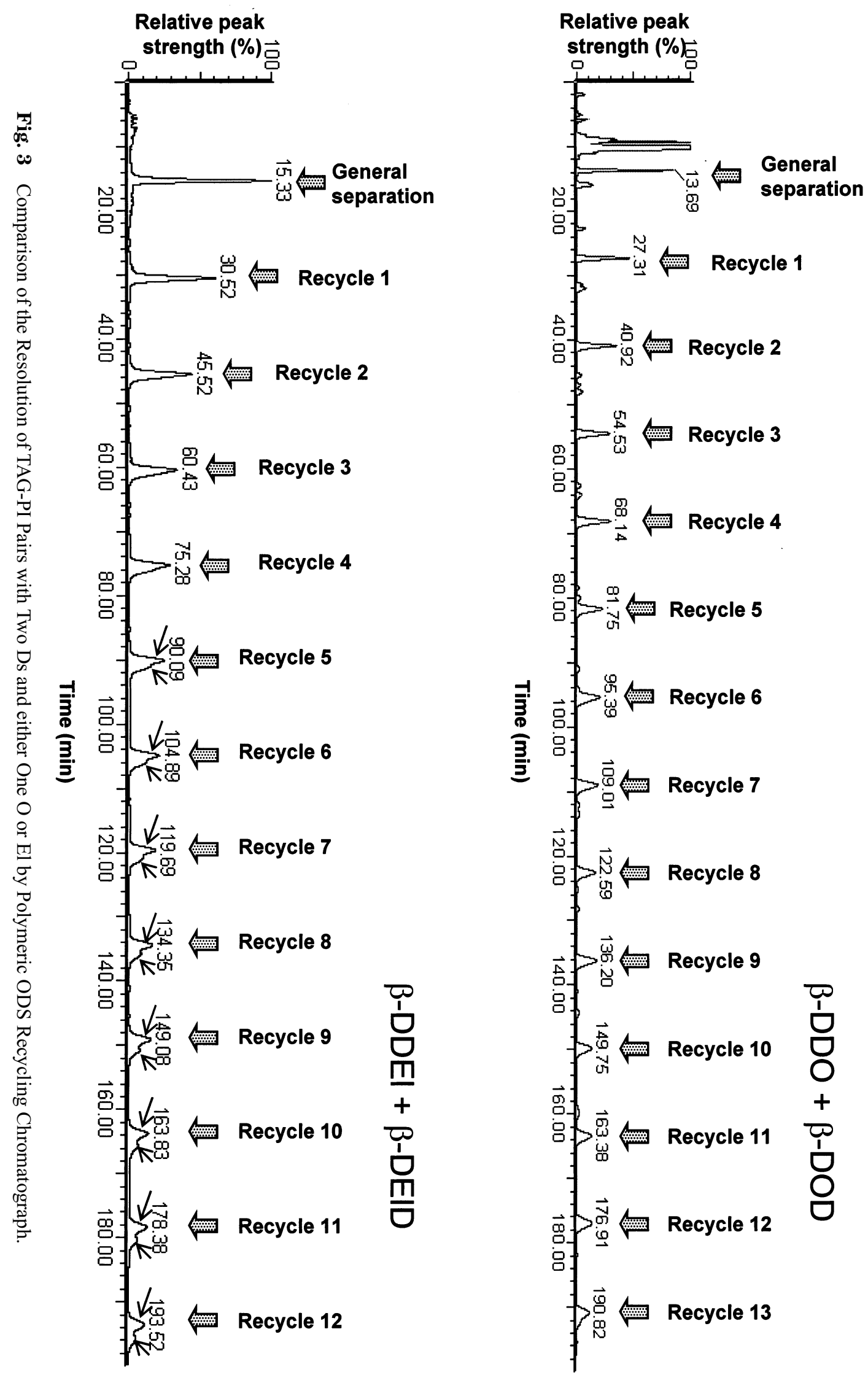
Table 2 Rs and TF Means for Pairs of TAG-PI Containing Two $\mathrm{C}_{8} \mathrm{~s}$ without Recycle Runs (n=3).

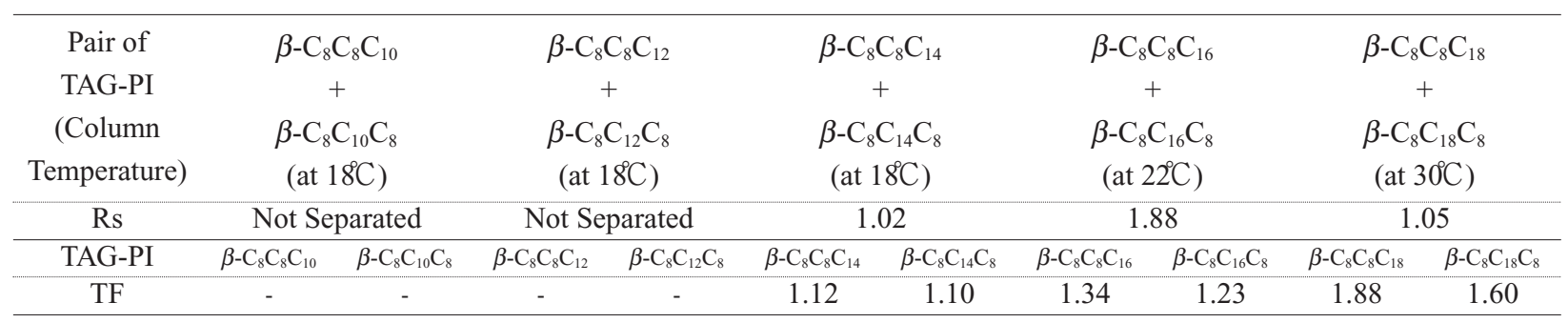

Table 3 Rs and TF Means for Pairs of TAG-PI Containing Two Ds without Recycle Runs ( $\mathrm{n}=3$ ).

\begin{tabular}{|c|c|c|c|c|c|c|c|c|c|}
\hline $\begin{array}{c}\text { Pair of } \\
\text { TAG-PI } \\
\text { (Column } \\
\text { Temperature) }\end{array}$ & $\begin{array}{c}\beta-\mathrm{DDC}_{10} \\
+ \\
\beta-\mathrm{DC}_{10} \mathrm{D} \\
(\text { at } 18 \mathrm{C} \text { ) }\end{array}$ & \multicolumn{2}{|c|}{$\begin{array}{c}\beta-\mathrm{DDC}_{12} \\
+ \\
\beta-\mathrm{DC}_{12} \mathrm{D} \\
\text { (at } 18 \mathrm{C} \text { ) }\end{array}$} & \multicolumn{2}{|c|}{$\begin{array}{c}\beta-\mathrm{DDC}_{14} \\
+ \\
\beta-\mathrm{DC}_{14} \mathrm{D} \\
(\text { at } 18 \mathrm{C} \text { ) }\end{array}$} & \multicolumn{2}{|c|}{$\begin{array}{c}\beta-\mathrm{DDC}_{16} \\
+ \\
\beta-\mathrm{DC}_{16} \mathrm{D} \\
\left(\text { at } 22^{\circ} \mathrm{C}\right)\end{array}$} & \multicolumn{2}{|c|}{$\begin{array}{c}\beta-\mathrm{DDC}_{18} \\
+ \\
\beta-\mathrm{DC}_{18} \mathrm{D} \\
\left(\text { at } 30^{\circ} \mathrm{C} \text { ) }\right.\end{array}$} \\
\hline Rs & Not Separated & \multicolumn{2}{|c|}{0.22} & \multicolumn{2}{|c|}{1.82} & \multicolumn{2}{|c|}{2.11} & \multicolumn{2}{|c|}{1.36} \\
\hline TAG-PI & $\beta-\mathrm{DDC}_{10}$ & $\beta-\mathrm{DDC}_{12}$ & $\beta-\mathrm{DC}_{12} \mathrm{D}$ & $\beta-\mathrm{DDC}_{14}$ & $\beta-\mathrm{DC}_{14} \mathrm{D}$ & $\beta-\mathrm{DDC}_{16}$ & $\beta-\mathrm{DC}_{16} \mathrm{D}$ & $\beta-$ DDC $_{18}$ & $\beta-\mathrm{DC}_{18} \mathrm{D}$ \\
\hline $\mathrm{TF}$ & - & 1.03 & 1.10 & 1.73 & 1.42 & 2.17 & 1.53 & 1.97 & 2.00 \\
\hline
\end{tabular}

\section{DISCUSSION}

Resolution of TAG-PIs comprising three saturated fatty acids was first examined to evaluate the effect of chain length on the resolution of TAG-PI pairs on an ODS-P column. Resolution was affected by the length of the saturated fatty acid chain. At least 14-carbon chain length was required for the complete separation of TAG-PI pairs without recycling. On the other hand, TAG-PI pairs consisting of two $\mathrm{C}_{8} \mathrm{~s}$ with one $\mathrm{C}_{10}$ or two $\mathrm{C}_{8} \mathrm{~s}$ with one $\mathrm{D}$ were not separated, even by several times of recycle runs. The same tendency was observed in TAG-PI pairs containing two Ds instead of the two $\mathrm{C}_{8}$ (Fig.2, Table 3). The recycle HPLC system automatically re-injects the elute back into the same column after passing through detector. For this reason, it is possible to increase the resolution of the column without increasing the column length and pressure. Thus, it is likely that the TAG-PI pairs described above are essentially unresolved on the non-endcapped polymeric ODS stationary phase. Kuroda et al. discussed ${ }^{18)}$ the resolution mechanism of TAG-PI on a polymeric ODS stationary phase using the "slot model" advocated by Wise and Sander ${ }^{21)}$. That is, the ODS groups comprising the polymeric stationary phase must be arranged more closely to each other than those of other kinds of ODS stationary phases, and the space between the ODS group in polymeric ODS phase should be narrower than that in monomeric ODS phase. Saturated fatty acids have free rotative structure and would deeply go into those spaces, thereby should strongly interact with the polymeric ODS groups than the unsaturated fatty acids, which have more rigid and bulky structures. This interaction referred to as "slot model" must be the principle of structural discrimination between TAG-PI pairs. Kuroda et al. also showed that the resolution of TAG-PIs was affected by the mobile phase species ${ }^{18}$. For example, the mobile phase containing tetrahydrofuran lost the resolution ability. Fujita et al. studied on the solvation of ODS stationary phase and mobile phase ${ }^{22,23)}$. They showed that tetrahydrofuran solvated with ODS stationary phase well, but methanol, ethanol, and 1-propanol did not. Probably, ODS stationary phase mixed with mobile phase containing tetrahydrofuran would swell and each ODS group might stand upward. As the result, the narrow space between the polymeric ODS stationary phase might have disappeared and the resolution ability for the pair of TAGPI might have lost. In contrast, a mixture of acetonitrile and 2-propanol $(6: 4, \mathrm{v} / \mathrm{v})$ was used as the mobile phase in this study and the solvation abilities of these organic solvents against ODS stationary phase are poor. Thus, the narrow space on the polymeric ODS stationary phase was kept and the pair of TAG-PI might be separated by the recognition of saturated fatty acids in TAG-PI. As demonstrated in the present study, the penetration depth in a polymeric ODS stationary phase might also be a very important factor. Particularly, myristic acid $\left(\mathrm{C}_{14}\right)$ or a chain of more than 14 carbons seems to be optimal for good resolution of TAG-PI (Table 2, 3). These chain lengths might be suitable for penetration into the polymeric ODS stationary phase. The peak symmetry of TAG-PI was also affected by chain length and decreased with an increase in the carbon chain length. The difference in peak symmetry might be due to the solubility of each TAG-PI in the mobile phase, their crystallinity, intermolecular association characteris- 


\section{N. Gotoh, Y. Matsumoto, H. Yuji et al.}

tics, the structures and conformation of the two fatty acids around the saturated fatty acid on the same glycerol backbone, etc. Separation of TAG-PI pairs containing $\mathrm{C}_{16}$ or $\mathrm{C}_{18}$ was performed at $22^{\circ} \mathrm{C}$ or $30^{\circ} \mathrm{C}$, respectively, because the peak shapes were fairly broad when separation was performed at $18^{\circ} \mathrm{C}$ (data not shown). It is likely that the solubility of the TAG-PI in the mobile phase and the TAG-PI melting point are more important factors for peak shape in this case than chain length for penetration into the polymeric ODS stationary phase. Based on the present results, the resolution of TAG-PI pairs is achieved by the recognition of the binding position of the saturated fatty acid on the glycerol backbone, and unsaturated fatty acids are not involved in this recognition because TAG-PI comprising two $\mathrm{C}_{8} \mathrm{~S}$ and one D (Fig. 1) or two Ds and one O (Fig. 3) were not separated. Normally, the separation of molecules by a reverse phase columns due to the recognition of the polar differences in the molecules. It seems likely, however, that the separation mechanism of a polymeric ODS phase differs for TAG-PI. Until now, the separation of TAG enantiomers could be achieved. Iwasaki et al. used chain length difference to resolve TAG enantiomers comprising two $\mathrm{C}_{8} \mathrm{~S}$ and one $\mathrm{D}$ with a chiral column ${ }^{24}$. The difference in the fatty acid chain length in TAG makes the recognition of TAG optical isomers easy, but TAG-PI comprising two $\mathrm{C}_{8} \mathrm{~S}$ and one D could not separated on a polymeric ODS stationary phase. This suggests that the polymeric ODS stationary phase does not recognize the difference of the whole TAG molecular structure. The stationary phase might recognize differences in the binding positions of the saturated fatty acids on the glycerol backbone. TAG-PIs comprising three unsaturated fatty acids were also examined. Pairs of TAG-PI comprising two Ds with one $O$ were not separated, may be owing to the lack of fatty acids with a rotative structure in the TAG molecules. It is noteworthy that the TAG-PI pair comprising two Ds with one O was not separated, but the TAG-PI pair comprising two Ds and one El could be separated using a recycle system. The difference between $\mathrm{O}$ and $\mathrm{El}$ is the structure of the double bond, i.e., $\mathrm{O}$ possesses a cis-type double bond and El possesses a trans-type double bond at the same position in the structure. The different double bond structure affects the whole molecular structure of the fatty acid, i.e., $\mathrm{O}$ is a bulky rigid structure, while $\mathrm{El}$ is a compact rigid structure. Probably, O cannot go into the space between the ODS group in polymeric ODS phase because of the bulky structure, whereas El probably can go into the space to some extent. The structure of TAG-PI comprising two Ds and one $\mathrm{C}_{18}$ is similar to that of TAG-PI comprising two Ds and one El, because both $\mathrm{El}$ and $\mathrm{C}_{18}$ consist of a linear 18-carbon chain. As mentioned above, the peak shape of a separated TAG-PI pair comprising two Ds and one $\mathrm{C}_{18}$ was broad at $18^{\circ} \mathrm{C}$, but they could be separated without a recycle run. Thus, $\mathrm{C}_{18}$ in TAG-PI completely goes into the space of a polymeric ODS stationary phase, allowing for separation without a recycle run. In contrast, however, the partially rigid structure owing to the trans double bond in El should have inhibited complete recognition by the polymeric ODS stationary phase. The space in the polymeric ODS stationary phase is very narrow and for this reason, El might not be allowed to penetrate enough to appear as two separate peaks on the chromatogram. These findings and explanations support the idea that the stationary phase recognizes differences in the saturated fatty acid structure and its binding position on the glycerol backbone to achieve the separation of a TAG-PI pair. It is also possible that the TAG-PI melting point and solubility in the mobile phase affect the resolution. Since we did not obtain data regarding the melting point and solubility of the TAG-PI pairs in the present study, we cannot discuss the TAG-PI separation from this aspect. This will be our future study.

Momchilova et al. reported the separation of TAG-PI pairs using a non-endcapped ODS column ${ }^{16,17)}$ such as that used in the present study. The role of the non-endcapped residual silanol group in the separation of TAG-PI was not addressed in their study, but the residual silanol group on the ODS stationary phase might be an important factor for TAG-PI separation because TAG-PI pairs cannot be separated on an endcapped polymeric ODS column (unpublished data). The findings of the present study indicate that TAG must contain saturated fatty acids possessing at least 12 carbons for separation on a non-endcapped polymeric ODS stationary phase. This method might be a useful and simple method for measuring the abundance ratio of TAGPI containing saturated fatty acids in natural oils.

\section{References}

1. Murray, R.K.; Granner, D.K.; Rodwell, V.W. Harper's Illustrated Biochemistry. $27^{\text {th }}$ ed. McGraw-Hill Companies. New York. p.124 (2006).

2. Owen R.F. Food Chemistry. $3^{\text {rd }}$ ed. Marcel Dekker Inc. New York. p. 231 (1996).

3. Murray, R.K.; Granner, D.K.; Rodwell, V.W. Harper's Illustrated Biochemistry. $27^{\text {th }}$ ed. McGraw-Hill Companies. New York. p.483 (2006).

4. Hunter, J.E. Studies on effect of dietary fatty acids as related to their position on triglycerides. Lipids 36, 655-668 (2001).

5. Kubow, S. The influence of positional distribution of fatty acids in native, interesterified and structure-specific lipids on lipoprotein metabolism and atherogenesis.

6. Sato, K. Crystallization behaviour of fats and lipids -a review-. Chem. Eng. Sci. 56, 2255-2265 (2001).

7. Fevrier, P.; Binet, A.; Dufosse, L.; Gree, R.; Yvergnaux, F. Separation of glyceride positional isomers by silver 
ion chromatography. J. Chromatogr. A 923, 53-57 (2001).

8. Adolf, R.; List, G. Analysis of triglyceride isomers by silver-ion high-performance liquid chromatography. Effect of column temperature on retention times. $J$. Chromatogr. A 1046, 109-113 (2004).

9. Dugo, P.; Kumm, T.; Presti, M.L.; Chiofalo, B.; Salimei, E.; Fazio, A.; Cotroneo, A.; Mondello, L. Determination of triacylglycerols in donkey milk by using high performance liquid chromatography coupled with atmospheric pressure chemical ionization mass spectrometry. J. Sep. Sci. 28, 1023-1030 (2005).

10. Byrdwell, W.C. Modern methods for lipid analysis by liquid chromatography/mass spectrometry and related techniques. AOCS PRESS. Champaign (2005).

11. Byrdwell, W.C. Atmospheric pressure chemical ionization mass spectrometry for analysis of lipids. Lipids 36, 327-346 (2001).

12. Mottram, H.R.; Evershed, R.P. Structure analysis of triacylglycerol positional isomers using atmospheric pressure chemical ionization mass spectrometry. Tetrahedron Lett. 37, 8593-8596 (1996).

13. Mottram, H.R.; Woodbury, S.E.; Evershed, R.P. Identification of triacylglycerol positional isomers present in vegetable oils by high performance liquid chromatography/atmospheric pressure chemical ionization mass spectrometry. Rapid Commun. Mass Spectrom. 11, 1240-1252 (1997).

14. Mottram, H.R.; Crossman, Z.M.; Evershed, R.P. Regiospecific characterization of the triacylglyerols in animal fats using high performance liquid chromatography-atmospheric pressure chemical ionization mass spectrometry. Analyst 126, 1018-1024 (2001).

15. Jakab, A.; Jablonkai, I.; Frogracs, E. Quantification of the ratio of positional isomer dilinoleoyl-oleoyl glycerols in vegetable oils. Rapid Commun. Mass Spec- trom. 17, 2295-2302 (2003).

16. Momchilova, S.; Tsuji, K.; Itabashi, Y.; NikolovaDamyanova, B.; Kuksis, A. Resolution of triacylglycerol positional isomers by reversed-phase high-performance liquid chromatography. J. Sep. Sci. 27, 10331036 (2004).

17. Momchilova, S.; Itabashi, Y.; Nikolova-Damyanova, B.; Kuksis, A. Regioselective separation of isomeric triacylglycerols by reversed-phase high-performance liquid chromatography: Stationary phase and mobile phase effects. J. Sep. Sci. 29, 2578-2583 (2006).

18. Kuroda, I.; Nagai, T.; Mizobe, H.; Yoshimura, N.; Gotoh, N.; Wada, S. HPLC separation of triacylglycerol positional isomers on a polymeric ODS column. Anal. Sci. 24, 865-869 (2008).

19. Reich, E.; Schibli, A. High-performance thin-layer chromatography for the analysis of medical plants. Thieme. New York. p.31 (2006).

20. Song, D.; Wang, J. Modified resolution factor for asymmetrical peaks in chromatographic separation. $J$. Pharm. Biomed. Anal. 32, 1105-1112 (2003).

21. Wise, S.A.; Sander, L.C. Factors affecting the reversedphase liquid chromatographic separation of polymeric aromatic hydrocarbon isomers. J. High Resolution Chromatogr. Chrom. Comm. 8, 248-255 (1985).

22. Fujita, N.; Nagae, N. A study of solvation phenomenon between organic solvent molecules in mobile phase and stationary phase on reversed-phase HPLC. Chromatography 26 Supplement 1, 21-24 (2005).

23. http://www.chromanik.co.jp/ligand_collapse1.html (as of 30 July 2009).

24. Iwasaki, Y.; Yasui, M.; Ishikawa, T.; Irimescu, R.; Hata, K.; Yamane, T. Optical resolution of asymmetric triacylglycerols by chiral-phase high-performance liquid chromatography. J. Chromatogr. A 905, 111-118 (2001). 